\title{
Efeitos da cirurgia bariátrica nos resultados perinatais em gestações classificadas como alto risco
}

\begin{abstract}
RESUMO | Objetivo: Analisar os desfechos maternos e perinatais em gestantes pós cirurgia bariátrica e classificadas como alto risco. Método: Trata-se de um estudo epidemiológico, observacional e retrospectivo, com abordagem quantitativa, realizado a partir de dados secundários de 4.293 gestantes de alto risco de um ambulatório especializado do sul do Brasil. Desse total, 50 gestantes tinham como condição clínica pré-existente a cirurgia bariátrica (grupo caso), e diante das características de idade, situação conjugal e escolaridades homogeneizou-se o grupo controle para comparação no estudo, obtendo-se 150 gestantes. A variável independente será a condição clínica pré-existente de cirurgia bariátrica. As variáveis desfechos serão: prematuridade, baixo peso ao nascer, Apgar $<7$ no $1^{\circ}$ e $5^{\circ}$ minutos, óbito fetal, óbito neonatal e tipo de parto. Resultados: Ao comparar o grupo controle com gestantes que possuem cirurgia bariátrica previa, notou-se um risco relativo aumentado para índice de Apgar abaixo de 7 no $1^{\circ}$ minuto. Conclusão: Com base na análise dos dados obtidos, conclui-se que a cirurgia bariátrica como condição clínica pré-existente em gestantes de alto risco não influencia negativamente os resultados perinatais. Todavia o acompanhamento durante pré-natal e puerpério devem ser realizados por uma equipe multidisciplinar e capacitada, que reconheça precocemente qualquer possível complicação.
\end{abstract}

Palavras-chaves: Saúde Materno-Infantil; Complicações na Gravidez; Cirurgia Bariátrica.

ABSTRACT | Objective: To analyze maternal and perinatal outcomes in pregnant women after bariatric surgery and classified as high risk. Method: This is an epidemiological, observational, and retrospective study, with a quantitative approach, based on secondary data from 4,293 high-risk pregnant women at a specialized outpatient clinic in southern Brazil. Of this total, 50 pregnant women had bariatric surgery as a pre-existing condition (case group), and given the characteristics of age, marital status and schooling, the control group was homogenized for comparison in the study, resulting in 150 pregnant women. The independent variable will be the pre-existing clinical condition of bariatric surgery. The outcome variables will be prematurity, low birth weight, Apgar $<7$ in the 1st and 5th minutes, fetal death, neonatal death and type of delivery. Results: When comparing the control group with pregnant women who had previous bariatric surgery, there was an increased relative risk for Apgar score below 7 in the 1st minute. Conclusion: Based on the analysis of the data obtained, it is concluded that bariatric surgery as a pre-existing clinical condition in high-risk pregnant women does not negatively influence perinatal results. However, prenatal and puerperium follow-up must be carried out by a multidisciplinary and trained team that recognizes any possible complications early.

Keywords: Maternal and Child Health; Pregnancy Complications; Bariatric Surgery.

RESUMEN | Objetivo: analizar los resultados maternos y perinatales en mujeres embarazadas después de la cirugía bariátrica y clasificarlos como de alto riesgo. Método: Este es un estudio epidemiológico, observacional y retrospectivo, con un enfoque cuantitativo, basado en datos secundarios de 4,293 mujeres embarazadas de alto riesgo en una clínica ambulatoria especializada en el sur de Brasil. De este total, 50 mujeres embarazadas se sometieron a cirugía bariátrica como una condición preexistente (grupo de casos), y dadas las características de edad, estado civil y educación, el grupo de control se homogeneizó para comparación en el estudio, lo que resultó en 150 mujeres embarazadas. La variable independiente será la condición clínica preexistente de la cirugía bariátrica. Las variables de resultado serán: prematuridad, bajo peso al nacer, Apgar $<7$ en el primer y quinto minutos, muerte fetal, muerte neonatal y tipo de parto. Resultados: Al comparar el grupo de control con mujeres embarazadas que se sometieron a cirugía bariátrica previa, hubo un mayor riesgo relativo de puntaje Apgar por debajo de 7 en el primer minuto. Conclusión: Con base en el análisis de los datos obtenidos, se concluye que la cirugía bariátrica como una condición clínica preexistente en mujeres embarazadas de alto riesgo no influye negativamente en los resultados perinatales. Sin embargo, el seguimiento prenatal y puerperio debe ser realizado por un equipo multidisciplinario y capacitado que reconozca cualquier posible complicación temprana. Descriptores: Salud Maternoinfantil; Complicaciones del Embarazo; Cirugía Bariátrica.

\section{Vinícius Dino Pozzebon}

Graduando em Medicina. Centro Universitário de Maringá (UNICESUMAR).

\section{Enzo Salles Fatuch}

Graduando em Medicina. Centro Universitário de Maringá (UNICESUMAR).

\section{Thaís Pordeus Leite Costa Mendes}

Médica. Especialista em Urgência e Emergência pela Faculdade Metropolitana do Vale do Aço (FAMEV).

Recebido em: 05/04/2020

Aprovado em: 08/04/2020

\section{Sandra Marisa Pelloso}

Enfermeira. Doutora em Enfermagem pela Universidade de São Paulo (USP). Docente do Programa de Pós Graduação em Ciências da Saúde e do Programa de Pós Graduação em Enfermagem da Universidade Estadual de Maringá (UEM).

\section{Marcos Benatti Antunes}

Enfermeiro. Doutor em Enfermagem pela Universidade Estadual de Maringá (UEM). Docente do Curso de Medicina do Centro Universitário de Maringá (UNICESUMAR).
INTRODUÇÃOO

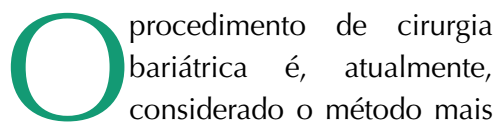
eficiente para o tratamento da obesidade e para a manutenção do peso a longo prazo. Dessa forma, com o aumento constante do número de pessoas obesas, junto a dificuldade do tratamento convencional (clínico) e aos bons resultados provenientes do procedimento cirúrgico, tem-se au- 
mentado significativamente o número de cirurgias bariátricas nos últimos anos ${ }^{(1)}$.

Contudo, há indicações para a realização deste procedimento, estipuladas pelo Ministério da Saúde (MS), tais como: para indivíduos que apresentam IMC (índice de massa corporal) $>50 \mathrm{~kg} / \mathrm{m}^{2}$; indivíduos com IMC $>40 \mathrm{~kg} / \mathrm{m}^{2}$ com ou sem comorbidades e sem sucesso no tratamento clinico realizado por no mínimo dois anos; e para indivíduos com IMC $>35 \mathrm{~kg} /$ $\mathrm{m}^{2}$ com comorbidades e sem sucesso no tratamento clínico, realizado também por no mínimo dois anos. Além disso, o MS também elenca critérios de indicação e de contraindicação para a cirurgia ${ }^{(2)}$.

Segundo a Sociedade Brasileira de Cirurgia Bariátrica e Metabólica (SBCBM), no ano de 2016, realizaram-se mais de 100.000 cirurgias bariátricas, e em $76 \%$ dos casos, o paciente era do sexo feminino. Dessa forma, o número de mulheres em idade fértil com cirurgia bariátrica prévia fica em aumento constante, e os cuidados relacionados às consequências do procedimento em futuras gestantes, ser maiores e realizados por uma equipe multiprofissional capacitada ${ }^{(3)}$.

Estes cuidados referem-se aos riscos do procedimento para mulheres gestantes, e dentre esses riscos, destacam-se as deficiências nutricionais que podem ocorrer após a cirurgia, causando déficits de vitaminas, proteínas e eletrólitos, e a suplementação inadequada no período de gestação pode provocar diversas alterações no feto, como retardo no crescimento e desequilíbrio eletrolítico, enfatizando novamente a função da equipe multiprofissional nesses cuidados, mais especificamente, da nutricionista neste caso; além disso, a possível má absorção provocada pela cirurgia bariátrica também pode gerar complicações fetais. Dessa maneira, a recomendação é para que a gestação ocorra após 18 meses da cirurgia bariátrica, afim de estabelecer novamente a homeostase na mulher ${ }^{(4)}$.

A gravidez é um evento fisiológico, natural, que normalmente ocorre sem
Segundo a Sociedade Brasileira de Cirurgia Bariátrica e Metabólica (SBCBM), no ano de 2016, realizaramse mais de 100.000 cirurgias bariátricas, e em $76 \%$ dos casos, o paciente era do sexo feminino. Dessa forma, o número de mulheres em idade fértil com cirurgia bariátrica prévia fica em aumento constante, e os cuidados relacionados às consequências do procedimento em futuras gestantes, ser maiores e realizados por uma equipe multiprofissional

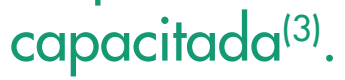

intercorrência, todavia, em cerca de $20 \%$ dos casos, existe a possibilidade da gravidez evoluir de forma desfavorável, tanto para a mãe, como para o feto, caracterizando uma gestação de alto risco. Esta, possui amplas condições obstétricas, sociais e clinicas, que podem causar complicações no período gestacional e que, por sua vez, podem comprometer o bem-estar do binômio materno-fetal, e levar ao desfecho desfavorável da gravidez. Essa avaliação de risco na gravidez é uma recomendação do MS, e consta com diversos fatores a serem considerados ${ }^{(5)}$.

Nesse contexto, o objetivo deste estudo foi analisar os desfechos maternos e perinatais em gestantes pós cirurgia bariátrica e classificadas como alto risco.

\section{METODOLOGIA}

Trata-se de um estudo epidemiológico, observacional e retrospectivo, com abordagem quantitativa, realizado a partir de dados secundários de 4.293 gestantes de alto risco de um ambulatório especializado do sul do Brasil. Desse total, 50 gestantes tinham como condição clínica pré-existente a cirurgia bariátrica (grupo caso), e diante das características de idade, situação conjugal e escolaridades homogeneizou-se o grupo controle para comparação no estudo, obtendo-se 150 gestantes.

Os dados foram coletados no período de novembro de 2016 e outubro de 2017 , por meio do prontuário da gestante, formulário de classificação de risco e livro de registro de recém-nascidos do hospital de referência ao parto.

A variável independente será a condição clínica pré-existente de cirurgia bariátrica. As variáveis desfechos serão: prematuridade, baixo peso ao nascer (BPN), Apgar $<7$ no $1^{\circ}$ e $5^{\circ}$ minutos, óbito fetal, óbito neonatal e cesárea.

Os dados foram digitados e organizados em uma planilha do Microsoft Office Excel $2017^{\circledR}$, posteriormente processamento e analisados com o softwa- 
re Epi Info 7.0, programa de domínio público. Os dados foram submetidos aos testes Qui-quadrado (x2) e Teste Exato de Fisher em nível de 5\% de significância $(p<0,05)$ e intervalo de confiança (IC) de 95\%.

Foram atendidas todas as normas de Pesquisas Envolvendo Seres Humanos do Conselho Nacional de Saúde (Resolução CNS n. ${ }^{\circ} 466 / 2012$ ) e foi aprovada conforme parecer $\mathrm{n} .^{\circ}$ 2.287.476 do Comitê Permanente de Ética em Pesquisa com Seres Humanos (COPEP).

\section{RESULTADOS}

As gestantes de alto risco que tem como condição clínica pré-existente a cirurgia bariátrica nesse estudo, apresentaram como características predominantes: idade entre 20 e 34 anos $(70,0 \%)$, pele branca $(82,0 \%)$; vivem com o companheiro $(70,0 \%)$ e escolaridade igual ou superior a 8 anos (76,0\%). Vale destacar que o grupo controle apresentou as mesmas características em idade, situação conjugal e escolaridades, pois foram critérios de homogeneização (Tabela 1).
Tabela 1.Perfil sociodemográfico das gestantes estratificadas como alto risco com antecedente obstétrico de Cirurgia Bariátrica e Grupo Controle. Maringá, PR, Brasil, 2017.

\begin{tabular}{|c|c|c|c|c|c|}
\hline \multirow[t]{2}{*}{ Variáveis } & \multicolumn{2}{|c|}{$\begin{array}{l}\text { Grupo Controle } \\
\qquad(n=150)\end{array}$} & \multicolumn{2}{|c|}{$\begin{array}{l}\text { Cirurgia Bariátrica } \\
\qquad(n=50)\end{array}$} & \multirow{2}{*}{$\mathrm{p}$} \\
\hline & $\mathrm{n}$ & $\%$ & $\mathrm{n}$ & $\%$ & \\
\hline Idade & & & & & 1,00 \\
\hline 20 a 34 & 105 & 70,0 & 35 & 70,0 & \\
\hline 35 ou mais & 45 & 30,0 & 15 & 30,0 & \\
\hline Cor & & & & & 0,40 \\
\hline Branca & 95 & 63,3 & 41 & 82,0 & \\
\hline Preta & 07 & 4,7 & 01 & 2,0 & \\
\hline Parda & 48 & 32,0 & 08 & 16,0 & \\
\hline Situação conjugal & & & & & 1,00 \\
\hline Vive com o companheiro & 105 & 70,0 & 35 & 70,0 & \\
\hline Não vive com o companheiro & 45 & 30,0 & 15 & 30,0 & \\
\hline Escolaridade & & & & & 1,00 \\
\hline$<8$ anos & 36 & 24,0 & 12 & 24,0 & \\
\hline$\geq 8$ anos & 114 & 76,0 & 38 & 76,0 & \\
\hline
\end{tabular}

Tabela 2. Análise da Cirurgia Bariátrica e grupo controle em relação aos resultados perinatais. Maringá, PR, Brasil, 2017.

\begin{tabular}{lcccccc} 
Resultados Perinatais & \multicolumn{3}{c}{$\begin{array}{c}\text { Grupo Controle } \\
(\mathrm{n}=150)\end{array}$} & \multicolumn{4}{c}{$\begin{array}{c}\text { Cirurgia Bariátrica } \\
(\mathrm{n}=50)\end{array}$} \\
& $\mathbf{n}$ & $\%$ & $\mathrm{n}$ & $\%$ & $\mathrm{RR}$ & $\mathrm{p}$ \\
\hline Prematuridade $(<37$ semanas $)$ & 60 & 40,0 & 13 & 26,0 & 0,81 & 0,07 \\
\hline Baixo peso ao nascer $(<2.500 \mathrm{~g})$ & 45 & 30,0 & 11 & 22,0 & 0,90 & 0,27 \\
\hline Apgar $1^{\circ}$ min. $(<7)$ & 38 & 25,3 & 05 & 10,0 & 0,83 & 0,01 \\
\hline Apgar $5^{\circ}$ min. $(<7)$ & 10 & 6,7 & 01 & 2,0 & 0,95 & 0,19 \\
\hline Morte fetal & 02 & 1,33 & - & - & - & - \\
\hline Morte infantil & 06 & 4,0 & - & - & - & - \\
\hline Cesárea & 109 & 72,7 & 41 & 82,00 & 1,52 & 0,18
\end{tabular}

Quando comparado o grupo de gestantes que apresentaram cirurgia bariátrica como antecedente obstétrico com o grupo-controle, observou-se risco relativo aumentado apenas para índice de Apgar abaixo de 7 no $1^{\circ}$ minuto $(R R=0,83$, $\mathrm{p}=0,01$ ) (Tabela 2).

\section{DISCUSSÃO}

Diante do exposto, notou-se um risco relativo aumentado para índice de Apgar abaixo de 7 no $1^{\circ}$ minuto, entretanto, as outras variáveis analisadas, como prematuridade, baixo peso ao nascer, Apgar no $5^{\circ}$ minuto, morte fetal, morte neonatal e tipo de parto, não apresentaram valores estatísticos significativos.

O número de cirurgias bariátricas em mulheres que estão em idade fértil está aumentando, fato este, que preocupa os profissionais da saúde, visto que a literatura científica ainda é escassa e não esclarece suficientemente os riscos do procedimento para estas mulheres gestantes, havendo opiniões contraditórias, conforme estudos com casuísticas reduzidas. A exemplo, uma pesquisa, que explicitou que a gravidez no pós-operatório do procedimento de cirurgia bariátrica é segura, todavia algumas gestantes apresentaram quadros de anemia e perda de peso importantes ${ }^{(6)}$. Corroborando ainda, outro estudo, ao analisar 159.210 partos em que, 298 partos foram para pacientes após operações bariátricas, constatou que a cirurgia bariátrica prévia, embora seja um fator de risco independente para cesariana $(p<0,001)$, não está associada a resultados perinatais adversos ${ }^{(7)}$.

Contudo, apesar dos escassos resultados e estudos sobre o tema, a gestante que previamente realizou cirurgia bariátrica, por ter passado por um procedimento que altera o metabolismo corpóreo, deve tomar maiores precauções e cuidados. Dentro das precauções e cuidados, pode-se enfatizar o tempo após a cirurgia bariátrica e antes de engravidar, que, para alguns autores, deve 
esperar cerca de um ano após o procedimento para engravidar, visto que neste período ocorrerá a perda de peso de forma mais intensa, e passado desse período, o corpo da mulher se adaptará, buscando a homeostase corpórea, logo, a gravidez após este período se tornaria mais segura ${ }^{(4)}$.

Nesse estudo, observou-se o índice de Apgar abaixo de 7 no primeiro minuto, como resultado estatístico significativo, contudo, outras alterações não obtiveram significância. Todavia, um estudo associou a obesidade a desfechos perinatais desfavoráveis, tais como risco aumentado de bebês com idade gestacional elevada, parto prematuro, diabetes gestacional, malformações congênitas, entre outros. Portanto, a obesidade, pode ocasionar complicações para a mulher e a futura criança, dessa forma a obesidade deve ser combatida, e em uma grande quantidade de casos, é combatida pela cirurgia bariátrica. Este procedimento, pode também, ocasionar complicações perinatais desfavoráveis ${ }^{(8)}$.

A cirurgia bariátrica, indutora da redução de peso e da atenuação dos riscos inerentes as doenças associadas a obesidade mórbida, apresenta relação com benefícios no âmbito reprodutivo, tanto para mulheres, como para homens ${ }^{(9)}$.

Os desfechos perinatais após o procedimento de cirurgia bariátrica tendem a se aproximar dos da população obstétrica em geral. Contudo, considerações especiais são necessárias ao cuidado de uma gravidez após o procedimento, e pesquisas adicionais devem considerar um papel maior da cirurgia bariátrica nos resultados perinatais em gestações ${ }^{(10)}$.

Segundo uma pesquisa, que avaliou dados bioquímicos de mulheres grávidas com cirurgia bariátrica previa, notou que, ao final da gravidez, que as mulheres apresentaram inadequações quanto à glicose, colesterol total, creatinina, zinco, albumina, triglicerídeos e vitamina D. Dessa forma, recomenda-se que a determinação do estado nutricional da gestante é importante, pois o período de gestação requer necessidades nutricionais específicas ${ }^{(11)}$.

Ao observar variações bioquímicas de mulheres após cirurgia bariátrica, entende-se que a gravidez segura só poderá ocorrer após a estabilização do peso corporal e das deficiências nutricionais causadas pelo procedimento, dessa forma, o aconselhamento antes da gravidez deve ser feito individualmente, com monitoramento e suplementação caso necessária, para que, quando a mulher com o procedimento prévio passe pelo período de gravidez, não encontre situações desfavoráveis ao binômio - mãe-bebêe $\hat{e}^{(12)}$.

O manual de assistência pré-natal da Federação Brasileira das Associações de Ginecologia e Obstetrícia (FEBRASGO), mulheres que são obesas tendem a possuem ciclos irregulares, e a perda de peso, por si só, pode levar ao aumento dos índices de fertilidade pós-operatório e normalizar os níveis hormonais da mulher, além de aumentar autoestima e ritmo de atividade sexual. Soma-se a isso, a diminuição dos riscos relacionados a hipertensão, pré-eclâmpsia, acidentes tromboembólicos, macrossomia fetal e diabetes, como consequência da redução do peso corporal da paciente, culminando na redução de possíveis complicações para o binômio materno-fetal ${ }^{(13)}$.

Outro estudo, demonstrou que pacientes obesas submetidas a gastroplastia (uma técnica de cirurgia bariátrica), apresentavam diminuição de significância na necessidade de cesárea, incidência de macrossomia fetal e diabetes gestacional. Todavia, o mesmo estudo explicita que as pacientes podem evoluir com deficiências de alguns compostos, tais como vitamina B12, folato, cálcio e ferro, podendo resultar, caso não ocorra o devido planejamento e acompanhamento, em aumento do risco para complicações maternas e fetais como anemia, restrição no crescimento intra-uterino e defeitos do tubo neural. Dessa forma, o estudo reitera a importância do acompanhamento frequente da gestante com cirurgia bariátrica prévia ${ }^{(14)}$.

Como já citado, gestantes submetidas previamente a cirurgia bariátrica possuem redução de riscos de complicações maternas (diabetes gestacional, hipertensão) e fetais (macrossomia fetal). Comparando-se o tipo de parto, há variações de índices de cesariana conforme a literatura, de 61,5 a 70\%. Com relação aos recém nascidos pequenos para a idade gestacional, também tem-se variações de 11,5 a 23,3\% ${ }^{(15,16)}$.

Em relação a prematuridade associada a cirurgia bariátrica, as análises desse estudo não apresentaram resultados significativos $(p=0,07)$, discordando, uma revisão sistemática com metanálise, apresenta os impactos das cirurgias bariátricas em resultados obstétricos e neonatais, e destaca a incidência de parto prematuro $(p=0,006)$, além disso, redução da incidência de pré-eclâmpsia $(p=0,007)$ e diabete gestacional $(p<$ $0,001)$, maior anemia materna $(p=0,002)$ e de admissão neonatal em centro de cuidados intensivos $-p=0,03^{(17)}$.

Vale ressaltar que, mulheres portadoras de obesidade possuem incidência três vezes maior de problemas ginecológicos, além disso, possuem alterações endócrinas e metabólicas como distúrbio em metabolismo de esteroide e alterações de secreção de hormônios, obtendo uma relação entre obesidade e infertilidade, através de hiperinsulinemia, hiperandrogenismo funcional e anovulação. Todos estes desfechos desfavoráveis a obesidade podem incentivar condições obstétricas, como qualidade ovocitária reduzida, pré-eclâmpsia, prematuridade, e outros. O papel do procedimento bariátrica é combater a obesidade, e acaba impactando positivamente em fertilidade e prognostico obstétrico dessas pacientes antes obesas. Todavia, mesmo com estes aspectos positivos, há recomendação entre tempo para gestação após o 
procedimento bariátrico, por conta das alterações que ocorrem no corpo da mulher ${ }^{(18)}$.

O consenso sobre o efeito de meIhora da fertilidade ocorre quando a redução de peso corporal atinge 10\%, e a cirurgia bariátrica é um meio eficiente de obter esta redução. Este mesmo estudo correlaciona que, cerca de $40 \%$ das mulheres analisadas no mesmo, não estavam cientes de que deveria esperar cerca de 12 a 18 meses para engravidar, a fim de reestabelecer a homeostase corporal. Este mesmo estudo demonstra que a obesidade influencia a fertilidade apenas antes dos 37 anos, e que após este período, o efeito da obesidade é superado pela idade, portanto, a perda de peso para melhora de fertilidade deve ocorrer antes dos 37 anos $^{(19)}$.

Mulheres submetidas a cirurgia bariátrica podem ter menor risco de complicações maternas, como diabetes ges- tacional, em comparação com obesas. Ademais, riscos de deficiência nutricional parece raro e baixo peso ao nascer não é totalmente claro. De fato, deve-se ter uma atenção diferenciada às mulheres que realizaram cirurgia bariátrica prévia para garantir nutrição materna e fetal adequada, além de reconhecer precocemente possíveis complicações, a exemplo tem-se a macrossomia fetal, ruptura prematura de membranas, morte perinatal e outras ${ }^{(20)}$.

Com base nos achados descritos e na análise da literatura científica, entende-se que a cirurgia bariátrica não influencia negativamente os resultados perinatais em gestações, de modo que pode apresentar em alguns casos pontos positivos da sua realização prévia. Todavia, a atenção a mulher gestante com cirurgia previa deve ser multiprofissional e cuidadosa, buscando reconhecer qualquer complicação com antecedência.

\section{CONCLUSÃO}

Com a análise dos resultados obstétricos e perinatais da população avaliada, conclui-se que a cirurgia bariátrica não influencia negativamente em resultados perinatais em gestações de alto risco, sendo, portanto, segura, desde que não seja precoce. É necessário enfatizar que o acompanhamento deve ser adequado e feito por uma equipe multiprofissional, garantindo um controle nutricional e pré-natal eficiente. Dessa forma, a gravidez, ao ocorrer no pós-operatório de cirurgia bariátrica, deve ocorrer com segurança. Contudo, investigações adicionais são necessárias para estabelecer de fato, as recomendações apropriadas referentes a este segmento de gestação, visto que estudos dessa natureza, apresentam limitações associadas, tais como casuística reduzida, não uniformidade de conclusões e escassas publicações.

\section{Referências}

1. Queiroz CS, Muniz LB, Haack $A$, Santos $A C D$. Gestação após cirurgia bariátrica: Um estudo baseado em evidências. REVISA. 2013; 2(1): 6572.

2. Ministério da Saúde (BR). Portaria n. ${ }^{\circ}$ 424, de 19 de março de 2013. Redefine as diretrizes para a organização da prevenção e do tratamento do sobrepeso e obesidade como linha de cuidado prioritária da Rede de Atenção à Saúde Pública das Pessoas com Doenças Crônicas. Diário Oficial da União, 2013.

3. Sociedade Brasileira de Cirurgia Bariátrica e Metabólica (BR). Número de cirurgias bariátricas no Brasil cresce 7,5\% em 2016. São Paulo, 2017. 4. Andreassen MS, Ferraz LF, Jesus SNR, Piano A, Azevedo CH, Perez AIC. Avaliação do binômio materno fetal após cirurgia bariátrica. BEPA, Bol. epidemiol. paul. (Online). 2012; 9(102): 21-29.

5. Rodrigues ARM, Dantas SLC, Pereira AMM, Silveira MAM, Rodrigues DF. Gravidez de alto risco: análise dos determinantes de saúde. SANARE. 2017; 16 Suppl 01: 23-28.

6. Dell Agnolo CM. Gravidez após cirurgia bariátrica: implicações para a mãe e o recém-nascido [dissertação]. Maringá (PR): Universidade Estadual de Maringá (UEM), 2009; 143p.

7. Sheiner R, Levy A, Silverberg D, Levy I, Katz M, Mazor M, et al. Pregnancy after bariatric surgery is not associated with adverse perinatal outcome. Am J Obstet Gynecol. 2004; 190(5): 1335-1340.

8. Johansson K, Cnattingius S, Näslund I, Roos N, Lagerros YT, Granath F, et al. Outcomes of pregnancy after bariatric surgery. N Engl J Med. 2015; 26(372):814-824.

9. Marques RR, Marques RR, Marques I. Gestação após cirurgia bariátrica: série de casos e revisão de literatura. Unoesc \& Ciência. 2016; 7(1):69-76.

10. Kominiarek MA. Preparing for and managing a pregnancy after bariatric surgery. Semin Perinatol. 2011; 35(6): 356-61.

11. Cunha CPV. Gravidez após Cirurgia Bariátrica: relação entre a ade- quação nutricional da gravida e os resultados neonatais. Porto: Universidade do Porto (UP), 2018.

12. González I, Lecube A, Rubio MA, García-Luna PP. Pregnancy after bariatric surgery: improving out-comes for mother and child. Internacional Journal of Women's Health. 2016; 8: 721.

13. Federação Brasileira das Associações de Ginecologia e Obstetrícia (BR). Manual de Assistência Pré-Natal. São Paulo: FEBRASGO, 2014.

14. Lima JG, Nóbrega LHC, Mesquita JB, Nóbrega MLC, Medeiros AC, Maranhão TCO et al. Gestação após gastroplastia para tratamento de obesidade mórbida: série de casos e revisão de literatura. Rev. Bras. Ginecol. Obstet. 2006; 28(2):107-111.

15. Nomura RMY, Dias MCC, Igai AMK, Liao LW, Miyadahira S, Zugaib M. Avaliação da vitalidade fetal e resultados perinatais em gestações após gastroplastia com derivação em Y de Roux. Rev. Assoc. Med. Bras. 2010; 56(6):670-674.

16.Patel JA, Patel NA, Thomas RL, Nelms JJC. Pregnancy outcomes after laparoscopic Roux-en-Y gastric bypass. Surg Obes Relat Dis. 2008; 4(1): 39-45.

17. Galazis N, Docheva N, Simillis C, Nicolaides KH. Maternal and neonatal outcomes in women under-going bariatric surgery: a systematic review and meta-analysis. Eur J Obstet Gynecol Reprod Biol. 2014; 181:45-53.

18. Melo FLE; Melo M. Impacto da cirurgia bariátrica na fertilidade feminina: revisão. Reprod Climat. 2017; 32(1):57-62.

19. Edison E, Whyte M, Vlymen J, Jones S, Lusignan S, Shawe J. Bariatric surgery in obese women of reproductive age improves conditions that underline fertility and pregnancy outcomes: retrospective cohort study of UK National Bariatric Surgery Registry (NBSR). Obes Surg. 2016; 26(12):2837-2842.

20. Hezelgrave NL, Oteng-Ntim E. Pregnancy after bariatric surgery: a re-view. J Obes. 2011; 2011:1-5. 